\title{
Multiband UWB System Performance with Random-Clustering Multipath-Rich Fading Channels
}

\author{
W. Pam Siriwongpairat, Weifeng $\mathrm{Su}^{\ddagger}$, and K. J. Ray Liu \\ Department of Electrical and Computer Engineering, University of Maryland, College Park, MD 20742 \\ ${ }^{\ddagger}$ Department of Electrical Engineering, State University of New York (SUNY) at Buffalo, Buffalo, NY 14260
}

\begin{abstract}
This paper provides a novel performance analysis for UWB systems that successfully captures the unique multipathrich property and multipath-clustering phenomenon of UWB channels. Using the Saleh-Valenzuela model, we characterize the pairwise error probability and the outage probability for UWB systems employing multiband OFDM based on the cluster arrival rate, the ray arrival rate within a cluster, and the cluster and ray decay factors. Furthermore, the effect of random-clustering phenomenon on the performance of UWB-MIMO systems is analyzed. The theoretical results reveal that regardless of the clustering behavior of UWB channels, the diversity gain can be improved by increasing the number of jointly encoded subcarriers, the number of jointly encoded OFDM symbols, or the number of antennas. The coding gain on the other hand, depends heavily on the cluster-arriving channels. Extensive simulation results are provided to support the theoretical analysis.
\end{abstract}

\section{INTRODUCTION}

The ultra large bandwidth of ultra-wideband (UWB) gives rise to important differences between UWB and narrowband channels, especially with respect to the number of resolvable paths and arrival times of multipath components [1]. In particular, the large bandwidth of UWB waveform considerably increases the ability of a receiver to resolve different reflections in UWB channels. As a result, the received signal contains a significant number of resolvable multipath components. Additionally, due to the fine time resolution of UWB waveform, the multipath components tend to occur in cluster rather than in a continuum, as is common for narrowband channels.

In recent years, a lot of research effort has been conducted in studying the performance of UWB systems (see [2] and references therein). However, most of the existing works are based on the stochastic tapped-delay-line (STDL) models [3] used in conventional narrowband/wideband systems. Performance analysis in STDL models is basically an extension of that for narrowband systems. More importantly, it does not reflect the multipath-rich nor random-clustering characteristics of UWB channels. To the best of our knowledge, none of the existing analysis is insightful in revealing the effect of the unique clustering characteristic on UWB system performances.

In order to implement an efficient UWB system, it is vital to capture the behavior of UWB channels, which has been characterized by the Saleh-Valenzuela $(\mathrm{S}-\mathrm{V})$ model [4]. In the $\mathrm{S}-\mathrm{V}$ model, the multipath components randomly arrive in cluster, and the arrival times are modeled by Poisson process. The multipath arrivals are grouped into cluster arrivals and ray

\footnotetext{
${ }^{\dagger}$ This work was supported in part by U. S. Army Research Laboratory under Cooperative Agreement DAAD 190120011.
}

arrivals within each cluster. The $\mathrm{S}-\mathrm{V}$ model is shown by the IEEE 802.15.3a Task Group [5] to best fit the realistic UWB channel measurements.

In this paper, we analyze the performance of the UWB systems that employ multiband orthogonal frequency division multiplexing (OFDM) [6] by taking into account the multipath-rich and random-clustering characteristics of UWB channels. Using the S-V model, we characterize the UWB performance in terms of cluster arrival rate, ray arrival rate, and cluster and ray decay factors. Two performance criteria we consider are pairwise error probability (PEP) and outage probability. First, we provide an exact PEP formulation for single-antenna multiband UWB systems. Then, we establish an approximation approach, which allows us to obtain a closed-form PEP formulation and an explicit outage probability expression. Finally, we generalize the performance results to space-time-frequency (STF) coded UWB multipleinput multiple-output (MIMO) systems. Simulation results are provided to support the theoretical analysis.

The remainder of this paper is organized as follows. A brief description of the channel and system models under consideration is given in Section II. In Section III, the PEP and outage probability analysis is provided. First, an approximation technique is established and a new closed-form PEP formulation is obtained. Then, we present closed-form expressions for the probability density function (PDF) and outage probability of the combined SNR over S-V fading scenarios. In Section IV, we characterize the performance of UWB-MIMO systems. Simulation results are presented in Section V, and some conclusions are drawn in Section VI.

\section{SySTEM MODEL}

We consider a peer-to-peer multiband OFDM system [6] as proposed in the IEEE 802.15.3a standard [5]. The multiband approach divides the available UWB spectrum into several subbands. Within each subband, the information is modulated using OFDM with $N$ subcarriers. The modulated OFDM symbols can be time-interleaved across different subbands [6].

\section{A. Channel Model}

The channel model specified in the IEEE 802.15.3a standard [1] is based on the S-V model for indoor channels [4]. In S-V model, the channel impulse response can be modeled by

$$
h(t)=\sum_{c=0}^{C} \sum_{l=0}^{L} \alpha(c, l) \delta\left(t-T_{c}-\tau_{c, l}\right),
$$


where $\alpha(c, l)$ denotes the gain of the $l^{\text {th }}$ multipath component in the $c^{t h}$ cluster, $T_{c}$ is the delay of the $c^{t h}$ cluster, and $\tau_{c, l}$ is the delay of the $l^{\text {th }}$ path in the $c^{\text {th }}$ cluster relative to the cluster arrival time. In (1), $C$ is the number of clusters, and $L$ is the number of paths within each cluster. The cluster arrivals and the path arrivals within each cluster can be modeled as Poisson distribution with rate $\Lambda$ and rate $\lambda(\lambda>\Lambda)$, respectively. The multipath gain coefficients $\alpha(c, l)$ are modeled as zero-mean, complex Gaussian random variables with variances [1]

$$
\Omega_{c, l}=\mathrm{E}\left[|\alpha(c, l)|^{2}\right]=\Omega_{0,0} \exp \left(-\frac{T_{c}}{\Gamma}-\frac{\tau_{c, l}}{\gamma}\right),
$$

where $\Omega_{0,0}$ is the mean energy of the first path of the first cluster, $\Gamma$ is the cluster decay factor, and $\gamma$ is the ray decay factor. The powers of the multipath components are normalized such that $\sum_{c=0}^{C} \sum_{l=0}^{L} \Omega_{c, l}=1$.

\section{B. Signal Model}

With the choice of cyclic prefix length greater than the duration of the channel impulse response, OFDM allows for each UWB subband to be divided into a set of $N$ orthogonal narrowband channels. At the transmitter, an information sequence is partitioned into blocks. Each block is mapped onto an $N \times 1$ matrix $\mathbf{D}=[d(0) d(1) \cdots d(N-1)]^{T}$, where $d(n), n=0,1, \ldots, N-1$, represents the complex channel symbol to be transmitted over subcarrier $n$. Suppose the information is jointly encoded across $M(1 \leq M \leq N)$ subcarriers. Particularly, the data matrix $\mathbf{D}$ is a concatenation of $P=\lfloor N / M\rfloor$ data matrices as follows:

$$
\mathbf{D}=\left[\begin{array}{lllll}
\mathbf{D}_{0}^{T} & \mathbf{D}_{1}^{T} & \cdots & \mathbf{D}_{(P-1)}^{T} & \mathbf{0}_{(N-P M) \times 1}
\end{array}\right]^{T},
$$

where $\mathbf{D}_{p}$ is an $M \times 1$ data matrix defined as $\mathbf{D}_{p}=$ $\left[\begin{array}{llll}d_{p}(0) & d_{p}(1) & \cdots & d_{p}(M-1)\end{array}\right]^{T}$ with $d_{p}(n) \triangleq d(p M+n)$ for $p=0,1, \ldots, P-1$, and $\mathbf{0}_{m \times n}$ stands for an $m \times n$ all-zero matrix. The data matrices $\mathbf{D}_{p}$ are independently designed for different $p$, and each data symbol $d_{p}(n)$ is normalized to have unit energy.

The received signal at the $n^{t h}$ subcarrier is given by

$$
y(n)=\sqrt{E_{s}} d(n) H(n)+z(n),
$$

where $E_{s}$ is the average transmitted energy per symbol, $H(n)$ is the channel frequency response, and $z(n)$ denotes the noise sample. We model $z(n)$ as zero-mean complex Gaussian random variable with variance $N_{0}$. The channel frequency response can be specified as

$$
H(n)=\sum_{c=0}^{C} \sum_{l=0}^{L} \alpha(c, l) \exp \left(-\mathbf{j} 2 \pi n \Delta f\left(T_{c}+\tau_{c, l}\right)\right),
$$

where $\mathbf{j} \triangleq \sqrt{-1}, \Delta f=1 / T$ is the frequency separation between two adjacent subcarriers, and $T$ is the OFDM symbol period. We assume that $H(n)$ is known at the receiver, but not at the transmitter.

\section{Performance Analysis}

In this section, we characterize the PEP and outage probability of multiband UWB systems in terms of multipath arrival rates and decay factors using the S-V fading model.

\section{A. Average PEP Analysis}

For subsequent performance evaluation, we format the received signal in (4) in a vector form as

$$
\mathbf{Y}_{p}=\sqrt{E_{s}} X\left(\mathbf{D}_{p}\right) \mathbf{H}_{p}+\mathbf{Z}_{p},
$$

where $X\left(\mathbf{D}_{p}\right)=\operatorname{diag}\left(d_{p}(0), d_{p}(1), \ldots, d_{p}(M-1)\right)$ is an $M \times M$ diagonal matrix with the elements of $\mathbf{D}_{p}$ on its main diagonal. In (6), the channel matrix $\mathbf{H}_{p}$, the received signal matrix $\mathbf{Y}_{p}$, and the noise matrix $\mathbf{Z}_{p}$ are of the same forms as $\mathbf{D}_{p}$ by replacing $d$ with $H, y$ and $z$, respectively. The receiver exploits a maximum likelihood decoder, where the decoding process is jointly performed within each data matrix $\mathbf{D}_{p}$, and the decision rule can be stated as

$$
\hat{\mathbf{D}}_{p}=\arg \min _{\mathbf{D}_{p}}\left\|\mathbf{Y}_{p}-\sqrt{E_{s}} X\left(\mathbf{D}_{p}\right) \mathbf{H}_{p}\right\|^{2},
$$

where $\|\cdot\|$ denotes the Frobenius norm [7].

Suppose that $\mathbf{D}_{p}$ and $\hat{\mathbf{D}}_{p}$ are two distinct data matrices. Following the computation steps as in [3], the average PEP, denoted as $P_{e}$, is given by

$$
P_{e}=\mathrm{E}\left[\mathrm{Q}\left(\sqrt{\frac{\rho}{2}\left\|\boldsymbol{\Delta}_{p} \mathbf{H}_{p}\right\|^{2}}\right)\right],
$$

where $\rho=E_{s} / N_{0}$ is the average signal-to-noise ratio (SNR), $\boldsymbol{\Delta}_{p}=X\left(\mathbf{D}_{p}\right)-X\left(\hat{\mathbf{D}}_{p}\right)$, and $\mathrm{Q}(x)$ is the Gaussian error function. Denoting

$$
\eta=\left\|\boldsymbol{\Delta}_{p} \mathbf{H}_{p}\right\|^{2}
$$

and using an alternate representation of $\mathrm{Q}$ function [8], $\mathrm{Q}(x)$ $=\frac{1}{\pi} \int_{0}^{\pi / 2} \exp \left(-\frac{x^{2}}{2 \sin ^{2} \theta}\right) d \theta$ for $x \geq 0$, the average PEP in (8) can be expressed as

$$
P_{e}=\frac{1}{\pi} \int_{0}^{\pi / 2} \mathcal{M}_{\eta}\left(-\frac{\rho}{4 \sin ^{2} \theta}\right) d \theta,
$$

where $\mathcal{M}_{\eta}(s)=\mathrm{E}[\exp (s \eta)]$ represents the moment generating function (MGF) of $\eta$ [8]. In general, it is difficult, if not possible, to determine $\mathcal{M}_{\eta}(s)$. However, for an uncoded multiband UWB system, i.e., when the number of jointly encoded subcarriers is $M=1$, we have the following result.

Theorem 1 When there is no coding across subcarriers, the average PEP of a UWB system employing multiband OFDM is given by

$$
P_{e}=\frac{1}{\pi} \int_{0}^{\pi / 2}\left(1+\frac{\rho}{4 \sin ^{2} \theta}|d-\hat{d}|^{2}\right)^{-1} d \theta
$$

\section{for any channel model parameters.}

The proof of Theorem 1 is omitted for simplicity. The above result reveals that the performance of an uncoded multiband UWB system does not depend on multipath arrival rates or decay factors, and it is the same as that of narrowband system in Rayleigh fading environment [8]. This implies that we cannot gain from the rich multipath-clustering property of UWB channels if the data is not encoded across subcarriers. 


\section{B. Approximate PEP Formulation}

We establish here an approximation which allows us to obtain an insightful understanding of the UWB systems when the information is jointly encoded across subcarriers.

Based on a representation of the quadratic form ([9], p.29), we can approximate $\eta$ as

$$
\eta \approx \sum_{n=1}^{M} \operatorname{eig}_{n}(\boldsymbol{\Phi})\left|\mu_{n}\right|^{2}
$$

where $\mu_{n}$ are iid zero-mean Gaussian random variables with unit variance, and

$$
\boldsymbol{\Phi}=\mathrm{E}\left[\boldsymbol{\Delta}_{p} \mathbf{H}_{p}\left(\boldsymbol{\Delta}_{p} \mathbf{H}_{p}\right)^{\mathcal{H}}\right]=\boldsymbol{\Delta}_{p} \mathbf{R}_{M} \boldsymbol{\Delta}_{p}^{\mathcal{H}},
$$

in which $\mathbf{R}_{M}=\mathrm{E}\left[\mathbf{H}_{p} \mathbf{H}_{p}^{\mathcal{H}}\right]$ is an $M \times M$ correlation matrix. Let the eigenvalues, $\operatorname{eig}_{n}(\boldsymbol{\Phi})$, be arranged in a non-increasing order as: $\operatorname{eig}_{1}(\boldsymbol{\Phi}) \geq \operatorname{eig}_{2}(\boldsymbol{\Phi}) \cdots \geq \operatorname{eig}_{M}(\boldsymbol{\Phi})$. By Ostrowski's theorem ([7], p.224), the eigenvalues of $\boldsymbol{\Phi}$ are given by

$$
\operatorname{eig}_{n}(\boldsymbol{\Phi})=\operatorname{eig}_{n}\left(\boldsymbol{\Delta}_{p} \mathbf{R}_{M} \boldsymbol{\Delta}_{p}^{\mathcal{H}}\right)=\nu_{n} \operatorname{eig}_{n}\left(\mathbf{R}_{M}\right),
$$

where $\nu_{n}$ is a nonnegative real number that satisfies $\operatorname{eig}_{M}\left(\boldsymbol{\Delta}_{p} \boldsymbol{\Delta}_{p}^{\mathcal{H}}\right) \leq \nu_{n} \leq \operatorname{eig}_{1}\left(\boldsymbol{\Delta}_{p} \boldsymbol{\Delta}_{p}^{\mathcal{H}}\right)$ for $n=1,2, \ldots, M$. From (12) and (14), we can approximate the MGF in (10) as

$$
\mathcal{M}_{\eta}(s) \approx \prod_{n=1}^{M} \frac{1}{1-s \nu_{n} \operatorname{eig}_{n}\left(\mathbf{R}_{M}\right)} .
$$

Now, the remaining problem is to determine the matrix $\mathbf{R}_{M}$. We observe that the $\left(n, n^{\prime}\right)^{t h}$ entry of the matrix $\mathbf{R}_{M}$ is $\mathrm{E}\left[H(n) H\left(n^{\prime}\right)^{*}\right]$ for $0 \leq n, n^{\prime} \leq M-1$. The elements on the main diagonal of $\mathbf{R}_{M}$ are given by

$$
R(n, n)=\mathrm{E}\left[|H(n)|^{2}\right]=\sum_{c=0}^{C} \sum_{l=0}^{L} \mathrm{E}\left[|\alpha(c, l)|^{2}\right]=1 .
$$

The off-diagonal elements of the matrix $\mathbf{R}_{M}, R\left(n, n^{\prime}\right)=$ $\mathrm{E}\left[H(n) H\left(n^{\prime}\right)^{*}\right]$ for $n \neq n^{\prime}$, can be evaluated as follows:

$R\left(n, n^{\prime}\right)=\sum_{c=0}^{C} \sum_{l=0}^{L} \mathrm{E}\left[\mathrm{E}\left[|\alpha(c, l)|^{2}\right] e^{-\mathbf{j} 2 \pi\left(n-n^{\prime}\right) \Delta f\left(T_{c}+\tau_{c, l}\right)}\right]$.

Let $R\left(n, n^{\prime}\right) \triangleq R\left(n-n^{\prime}\right)$, and substitute (2) into (17), yielding

$$
R(m)=\sum_{c=0}^{C} \sum_{l=0}^{L} \Omega_{0,0} \mathrm{E}\left[e^{-g\left(\frac{1}{\Gamma}, m\right) T_{c}-g\left(\frac{1}{\gamma}, m\right) \tau_{c, l}}\right],
$$

where $g(a, m) \triangleq a+\mathbf{j} 2 \pi m \Delta f$. Average (18) with respect to the Poisson distribution, after some manipulations, we arrive at

$$
R(m)=\Omega_{0,0} \frac{\Lambda+g\left(\frac{1}{\Gamma}, m\right)}{g\left(\frac{1}{\Gamma}, m\right)} \frac{\lambda+g\left(\frac{1}{\gamma}, m\right)}{g\left(\frac{1}{\gamma}, m\right)} .
$$

Finally, by substituting (15) into (10), we can characterize the multiband UWB performance as follows.

Theorem 2 When the information is jointly encoded across $M$ $(1 \leq M \leq N)$ subcarriers, the average PEP of a multiband $U W B$ system can be approximated as

$$
P_{e} \approx \frac{1}{\pi} \int_{0}^{\pi / 2} \prod_{n=1}^{M}\left(1+\frac{\rho \nu_{n}}{4 \sin ^{2} \theta} \operatorname{eig}_{n}\left(\mathbf{R}_{M}\right)\right)^{-1} d \theta,
$$

where the $M \times M$ matrix $\mathbf{R}_{M}$ is given by

$$
\mathbf{R}_{M}=\left(\begin{array}{cccc}
1 & R(1)^{*} & \cdots & R(M-1)^{*} \\
R(1) & 1 & \cdots & R(M-2)^{*} \\
\vdots & \vdots & \ddots & \vdots \\
R(M-1) & R(M-2) & \cdots & 1
\end{array}\right)
$$

and $R(m)$ for $m=1,2, \ldots, M$ are defined in (19).

It is worth noting that the result in Theorem 2 can be straightforwardly extended to the case when interleaving or permutation over different subcarriers is applied. To be specific, if the data matrix is permuted such that the data symbol $d_{p}(n)$ is transmitted in subcarrier $\sigma_{p}(n)$ instead of subcarrier $n$, then the PEP performance of the permuted data matrix is of the same form as (20) with the off-diagonal elements of matrix $\mathbf{R}_{M}$ replaced by $R\left(n, n^{\prime}\right)=R\left(\sigma_{p}(n)-\sigma_{p}\left(n^{\prime}\right)\right)$.

In the sequel, we discuss the PEP approximations in Theorem 2 for two special cases to get some insight.

1) In case of no coding, i.e., $M=1$, the PEP can be obtained from (20) as

$$
P_{e} \approx \frac{1}{\pi} \int_{0}^{\pi / 2}\left(1+\frac{\rho}{4 \sin ^{2} \theta}|d-\hat{d}|^{2}\right)^{-1} d \theta,
$$

which is consistent with the exact PEP given in (11).

2) In case of jointly encoding across 2 subcarriers, i.e., $M=2$, the PEP can be approximated by (20) as

$$
P_{e} \approx \frac{1}{\pi} \int_{0}^{\pi / 2}\left(1+\frac{\rho J+\rho^{2} \nu_{1} \nu_{2}\left(1-B^{2}\right)}{16 \sin ^{4} \theta}\right)^{-1} d \theta,
$$

where $J=4 \sin ^{2} \theta\left[\nu_{1}+\nu_{2}+B\left(\nu_{1}-\nu_{2}\right)\right]$ and

$$
B=\Omega_{0,0} \frac{\left[\left(\Lambda+\frac{1}{\Gamma}\right)^{2}+b\right]^{\frac{1}{2}}\left[\left(\lambda+\frac{1}{\gamma}\right)^{2}+b\right]^{\frac{1}{2}}}{\left[\left(\frac{1}{\Gamma}\right)^{2}+b\right]^{\frac{1}{2}}\left[\left(\frac{1}{\gamma}\right)^{2}+b\right]^{\frac{1}{2}}},
$$

and $b=(2 \pi \Delta f)^{2}$. In UWB system, $b$ is normally much less than $\frac{1}{\gamma^{2}}$ and $\frac{1}{\Gamma^{2}}$. Hence, (24) can be approximated by

$$
B \approx \Omega_{0,0}(\Lambda \Gamma+1)(\lambda \gamma+1) .
$$

The PEP in (20) reveals that the coded multiband UWB performance depends on the correlations in the frequency response among different subcarriers, $R(m)$, which in turn relate to the path arrival rates and decay factors. If the number of jointly encoded subcarriers is $M=2$, (23) shows that the performance is related to the channel model (CM) parameters through the factor $B$. For instance, under CM 1 and CM 4 [1], we have $B=0.9852$ and 0.8351 , respectively. The PEP in (23) reveals that UWB performance in CM 4 is better than that in $\mathrm{CM}$ 1. This comes from the fact that CM 4 contains more random multipath components, which implies less correlation in the channel frequency response, and hence yields better performance than CM 1. In some scenarios, e.g., a very shortrange line-of-sight environment [1], the product of the cluster arrival rate and cluster decay factor can be much less than one. In this case, $B \approx \Omega_{0,0}(\lambda \gamma+1)$ which implies that only the first cluster strongly affects the performance. 


\section{Outage Probability}

The outage probability [8] is defined as the probability that the combined SNR, $\zeta$, falls below a specified threshold, $\zeta_{o}$, namely $P_{\text {out }}=P\left(\zeta \leq \zeta_{o}\right)=\int_{0}^{\zeta_{o}} p_{\zeta}(x) d x$, where $p_{\zeta}(x)$ denotes the probability density function (PDF) of $\zeta$. Since the data is jointly en/decoded for each matrix $\mathbf{D}_{p}$, the combined SNR can be defined as

$$
\zeta=\frac{E_{s}\left\|X\left(\mathbf{D}_{p}\right) \mathbf{H}_{p}\right\|^{2}}{\mathrm{E}\left[\left\|\mathbf{Z}_{p}\right\|^{2}\right]}=\frac{\rho}{M} \sum_{n=0}^{M-1}\left|H_{p}(n)\right|^{2},
$$

in which $\rho=E_{s} / N_{0}$. Denote $\xi=\sum_{n=0}^{M-1}\left|H_{p}(n)\right|^{2}$, then the outage probability can be expressed as

$$
P_{\text {out }}=P\left(\xi \leq \frac{M \zeta_{o}}{\rho}\right)=\int_{0}^{\frac{M \zeta_{o}}{\rho}} p_{\xi}(x) d x .
$$

To determine the PDF $p_{\xi}(x)$, we first obtain the MGF of $\xi$ from the MGF $\mathcal{M}_{\eta}(s)$ by replacing $\boldsymbol{\Delta}_{p}$ with an identity matrix, and then apply the inverse Laplace transform to $\mathcal{M}_{\xi}(s)$. After some computations, we have the following results.

Theorem 3 When there is no coding across subcarriers, the outage probability of a multiband UWB system is given by

$$
P_{\text {out }}=1-e^{-\zeta_{o} / \rho}
$$

for any channel model parameters. When the information is jointly encoded across $M(1<M \leq N)$ subcarriers, the outage probability can be approximated as

$$
P_{\text {out }} \approx \sum_{n=1}^{M} A_{n}\left(1-e^{-\zeta_{o} M /\left(\rho \operatorname{eig}_{n}\left(\mathbf{R}_{M}\right)\right)}\right),
$$

where $\mathbf{R}_{M}$ is specified in (21), and $A_{n}$ is given by

$$
A_{n}=\prod_{n^{\prime}=1, n^{\prime} \neq n}^{M} \operatorname{eig}_{n}\left(\mathbf{R}_{M}\right)\left[\operatorname{eig}_{n}\left(\mathbf{R}_{M}\right)-\operatorname{eig}_{n^{\prime}}\left(\mathbf{R}_{M}\right)\right]^{-1} \text {. }
$$

The above analysis shows that the outage probability follows the same behaviors as the average PEP. Specifically, the outage probability of an uncoded multiband UWB system does not depend on the clustering property of UWB channel, and it is the same as that for narrowband Rayleigh fading environment [8]. When the data is jointly encoded across multiple subcarriers, (29) discloses that the outage probability is related to the eigenvalues of the correlation matrix $\mathbf{R}_{M}$, which depends on the path arrival rates and decay factors.

To gain some insightful understanding on the outage probability formulation in (29), let us consider a specific example of jointly encoding across $M=2$ subcarriers. In this case, the outage probability can be approximated as

$$
\begin{aligned}
P_{\text {out }} \approx & 1-0.5\left(1+B^{-1}\right) \exp \left(-\frac{2 \zeta_{o}}{\rho(1+B)}\right) \\
& -0.5\left(1-B^{-1}\right) \exp \left(-\frac{2 \zeta_{o}}{\rho(1-B)}\right),
\end{aligned}
$$

where $B$ is defined in (24). Since $B$ takes any value between 0 and $1(0<B<1)$, the higher the $B$ the larger the outage probability $P_{\text {out }}$ in (30). For instance, $B=0.9852$ in case of CM 1 and $B=0.8351$ in case of CM 4. As the SNR increases, $P_{\text {out }}$ for CM 4 drops faster than that for CM 1 due to the effect of the third term in $P_{\text {out }}$ expressions. Explicitly, the term $0.0987 \exp \left(-\frac{12.131 \zeta_{o}}{\rho}\right)$ for CM 4 increases with SNR $\rho$ much faster than the term $0.0075 \exp \left(-\frac{134.91 \zeta_{o}}{\rho}\right)$ for CM 1 . Hence, the outage probability performance for $\mathrm{CM} 4$ tends to be better than that for CM 1 at high SNR.

\section{ANALYSIS FOR UWB-MIMO SYSTEMS}

In this section, we describe a UWB-MIMO system model, and then characterize its PEP performances in the S-V model.

\section{A. UWB-MIMO System Model}

Consider a UWB-MIMO system with $N_{t}$ transmit and $N_{r}$ receive antennas. The channel impulse response from the $i^{t h}$ transmit antenna to the $j^{t h}$ receive antenna during the $k^{t h}$ OFDM block can be modeled in the same form as (1) with the multipath gain coefficient replaced by $\alpha_{i j}^{k}(c, l)$. We assume that the average powers $\Omega_{c, l}$ and the delays $T_{c}$ and $\tau_{c, l}$ are the same for every transmit-receive link.

At the transmitter, the information is jointly encoded across $N_{t}$ transmit antennas, $M$ OFDM subcarriers, and $K$ OFDM blocks. Each STF codeword can be expressed as a $K M \times N_{t}$ matrix $\mathbf{D}_{p}=\left[\left(\mathbf{D}_{p}^{0}\right)^{T}\left(\mathbf{D}_{p}^{1}\right)^{T} \cdots\left(\mathbf{D}_{p}^{K-1}\right)^{T}\right]^{T}$, where $\mathbf{D}_{p}^{k}=\left[\mathbf{d}_{p, 1}^{k} \mathbf{d}_{p, 2}^{k} \cdots \mathbf{d}_{p, N_{t}}^{k}\right]$ and $\mathbf{d}_{p, i}^{k}=\left[d_{i}^{k}(p M) d_{i}^{k}(p M+\right.$ 1) $\left.\cdots d_{i}^{k}(p M+M-1)\right]^{T}$ for $i \stackrel{p, i}{=} 1,2, \ldots, N_{t}$ and $k=$ $0,1, \ldots, K-1$. The symbol $d_{i}^{k}(n), n=0,1, \ldots, N$, represents the complex symbol to be transmitted over subcarrier $n$ by transmit antenna $i$ during the $k^{\text {th }}$ OFDM symbol period.

The received signal at the $n^{t h}$ subcarrier at receive antenna $j$ during the $k^{\text {th }}$ OFDM symbol duration can be expressed as

$$
y_{j}^{k}(n)=\sqrt{\frac{E_{s}}{N_{t}}} \sum_{i=1}^{N_{t}} d_{i}^{k}(n) H_{i j}^{k}(n)+z_{j}^{k}(n),
$$

where $H_{i j}^{k}(n)=\sum_{c=0}^{C} \sum_{l=0}^{L} \alpha_{i j}^{k}(c, l) e^{-\mathbf{j} 2 \pi n \Delta f\left(T_{c}+\tau_{c, l}\right)}$ is the channel frequency response, and $z_{j}^{k}(n)$ is the additive noise.

Due to the band hopping, the $K$ OFDM symbols in each STF codeword are sent over different subbands. With an ideal band hopping, we assume that the signals transmitted over $K$ different subbands undergo independent fading. For simplicity, the MIMO channel is assumed to be spatially uncorrelated.

\section{B. Pairwise Error Probability}

Similarly, the PEP between two distinct STF codewords $\mathbf{D}_{p}$ and $\hat{\mathbf{D}}_{p}$ can be given by

$$
P_{e}=\mathrm{E}\left[\mathrm{Q}\left(\sqrt{\frac{\rho}{2 N_{t}} \sum_{j=1}^{N_{r}}\left\|\boldsymbol{\Delta}_{p} \mathbf{H}_{p, j}\right\|^{2}}\right)\right],
$$

where $\boldsymbol{\Delta}_{p}=X\left(\mathbf{D}_{p}\right)-X\left(\hat{\mathbf{D}}_{p}\right)$, in which $X\left(\mathbf{D}_{p}\right)$ converts each column of $\mathbf{D}_{p}$ into a diagonal matrix and results in an $K M \times K M N_{t}$ matrix: $X\left(\mathbf{D}_{p}\right)=X\left(\left[\mathbf{d}_{p, 1} \cdots \mathbf{d}_{p, N_{t}}\right]\right)=$ $\left[\operatorname{diag}\left(\mathbf{d}_{p, 1}\right) \cdots \operatorname{diag}\left(\mathbf{d}_{p, N_{t}}\right)\right]$. In (32), $\mathbf{H}_{p, j}$ is a $K M N_{t} \times$ 1 channel matrix formatted as $\mathbf{H}_{p, j}=\left[\begin{array}{ll}\mathbf{H}_{p, 1 j}^{T} & \mathbf{H}_{p, 2 j}^{T}\end{array}\right.$ $\left.\cdots \mathbf{H}_{p, N_{t} j}^{T}\right]^{T}$, in which $\mathbf{H}_{p, i j}=\left[\begin{array}{llll}H_{i j}^{0}(p M) & \cdots & H_{i j}^{0} & (p M+\end{array}\right.$ 
$\left.M-1) \cdots H_{i j}^{K-1}(p M) \cdots H_{i j}^{K-1}(p M+M-1)\right]^{T}$. Following the same procedure as in single antenna transmission, we first obtain

$$
\eta=\sum_{j=1}^{N_{r}}\left\|\boldsymbol{\Delta}_{p} \mathbf{H}_{p}\right\|^{2} \approx \sum_{j=1}^{N_{r}} \sum_{n=1}^{M} \operatorname{eig}_{n}\left(\boldsymbol{\Phi}_{j}\right)\left|\mu_{j, n}\right|^{2},
$$

where $\boldsymbol{\Phi}_{j}=\boldsymbol{\Delta}_{p} \mathrm{E}\left[\mathbf{H}_{p, j} \mathbf{H}_{p, j}^{\mathcal{H}}\right] \boldsymbol{\Delta}_{p}^{\mathcal{H}}$, and $\mu_{j, n}$ are iid zeromean Gaussian random variables with unit variance. After some algebra, $\boldsymbol{\Phi}_{j}$ can be re-expressed as

$$
\boldsymbol{\Phi}_{j}=\left(\mathbf{D}_{p}-\hat{\mathbf{D}}_{p}\right)\left(\mathbf{D}_{p}-\hat{\mathbf{D}}_{p}\right)^{\mathcal{H}} \circ\left(\mathbf{I}_{K} \otimes \mathbf{R}_{M}\right),
$$

where $\mathbf{R}_{M}$ is specified in (21), $\otimes$ denotes the Kronecker product, and $\circ$ denotes the Hadamard product [7]. Define $\mathbf{S}_{k} \triangleq\left(\mathbf{D}_{p}^{k}-\hat{\mathbf{D}}_{p}^{k}\right)\left(\mathbf{D}_{p}^{k}-\hat{\mathbf{D}}_{p}^{k}\right)^{\mathcal{H}}$. Suppose the information is repeated over $K$ OFDM symbols, then $\mathbf{S}^{k}$ is the same for all $k$. Substituting (34) into (33) and using the MGF of $\eta$, the average PEP can be upper bounded at high SNR as

$$
P_{e} \lesssim \prod_{n=1}^{r}\left(\frac{\rho}{4 N_{t}} \operatorname{eig}_{n}\left(\mathbf{S}_{0} \circ \mathbf{R}_{M}\right)\right)^{-K N_{r}},
$$

which implies a diversity order of $r K N_{r}$ and a coding gain of $\frac{1}{4 N_{t}}\left(\prod_{n=1}^{r} \operatorname{eig}_{n}\left(\mathbf{S}_{0} \circ \mathbf{R}_{M}\right)\right)^{1 / r}$, where $r$ denotes the rank of matrix $\mathbf{S}_{0} \circ \mathbf{R}_{M}$. Since UWB channel contains a large number of resolvable paths, $\mathbf{R}_{M}$ is generally of full rank. This leads to an interesting observation that the multiband UWB system achieves the same diversity advantage in different channel environment. Only the system coding gain that depends heavily on the cluster arriving fading paths. For example, when $N_{t}=2, M=4$, and the STF codeword is based on a repetition code [10] with Alamouti's structure [11], then the PEP can be determined as

$$
P_{e} \lesssim\left(\frac{\rho \nu}{8} \sqrt{1-\Omega_{0,0}^{2}(\Lambda \Gamma+1)^{2}(\lambda \gamma+1)^{2}}\right)^{-4 K N_{r}},
$$

where $\nu \triangleq \sum_{i=1}^{2}\left|d_{i}-\hat{d}_{i}\right|^{2}$. Clearly, the diversity gain is $4 K N_{r}$ for each channel model, whereas the coding gain is about $0.0214 \nu$ for CM 1 and $0.0688 \nu$ for CM 4 . Such coding advantage makes the performance of multiband UWB system under $\mathrm{CM} 4$ superior to that under CM 1.

\section{Simulation Results}

We performed simulations for a multiband UWB system with $N=128$ subcarriers, the subband bandwidth of 528 $\mathrm{MHz}$, and QPSK signals. The channel model parameters followed those for CM 1 and CM 4 [1]. The data symbols are selected from QPSK constellations. In our simulations, the data matrix $\mathbf{D}_{p}$ were constructed via a repetition mapping [10], and Alamouti's structure was used for UWB-MIMO system.

Figs. 1 and 2 depict the average symbol-error-rate (SER) performances of single-antenna multiband UWB system as functions of average SNR per bit $\left(E_{b} / N_{0}\right)$ in $\mathrm{dB}$. Note that we used the union bound [8] to obtain the average SER from the PEP formulation. In Fig. 1, we show the simulated and theoretical performances of uncoded multiband UWB system $(M=1)$. We observe that the performances of UWB system in CM 1 and CM 4 are almost the same, and they are close to the exact PEP calculation in (11). The simulation results

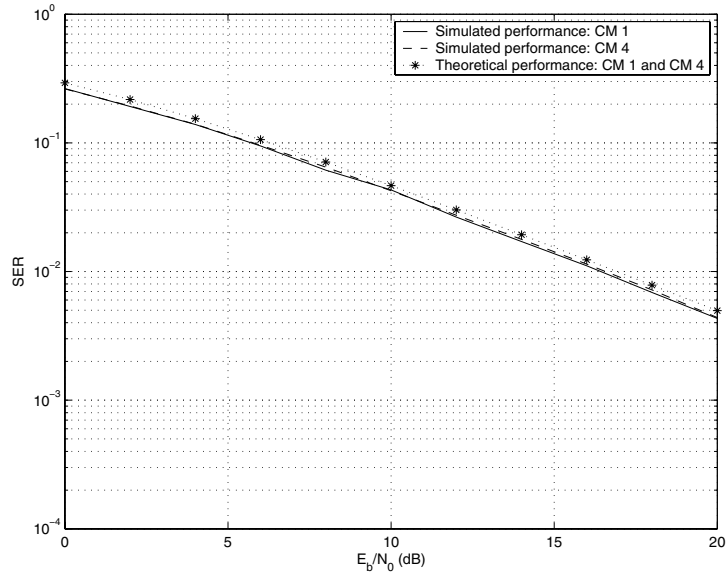

Fig. 1: PEP of uncoded multiband UWB system

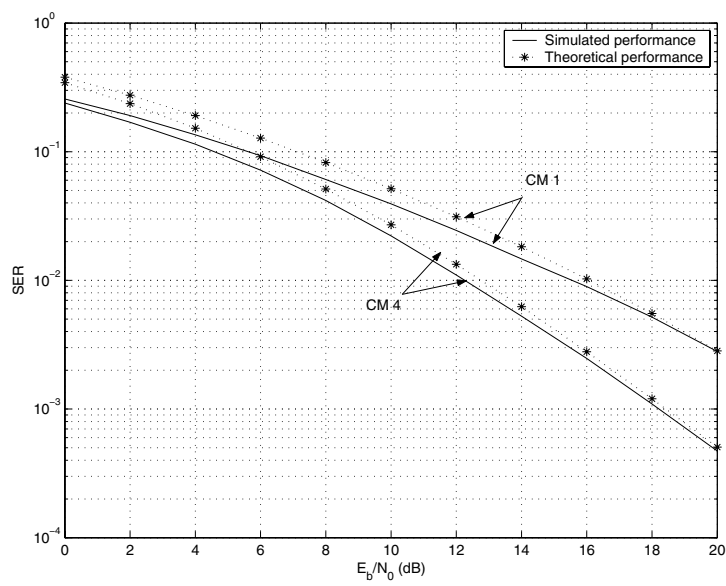

Fig. 2: PEP of coded multiband UWB system

confirm the theoretical expectation that the performances of multiband UWB systems without coding across subcarriers are the same for different environments. Fig. 2 shows the UWB performances when the information is jointly encoded across two subcarriers $(M=2)$. The theoretical approximations obtained from (20) are close to the simulated performances for both CM 1 and CM 4. In addition, the performance obtained under $\mathrm{CM} 4$ is superior to that under $\mathrm{CM} 1$, which is in agreement with the theoretical results in Section III-B.

Figs. 3 (a) and (b) plot the outage probability $P_{\text {out }}$ versus normalized average SNR $\rho / \zeta_{o}$ in $\mathrm{dB}$. We can observe that the outage probability follows the same tendencies as the average SER. The uncoded system yields the same outage probability in both CM 1 and CM 4, whereas the coded system under CM 4 achieves a lower outage probability, hence better performance, than that with $\mathrm{CM} 1$.

Figs. 4 (a) and (b) depict the SER performances for UWBMIMO system with the information jointly encoded across $N_{t}=2$ transmit antennas, $K=1$ OFDM symbol, and $M=$ 2,4 subcarriers. Note that the theoretical SER was obtained from the union bound of the PEP formulation in (35). From both figures, we can see that the theoretical approximation in (35) correctly predicts the diversity and coding gains. From Fig. 4 (b), it is clear that the multiband system under CM 4 outperforms that under CM 1 due to the larger coding gain. Fig. 4 confirms our observation in Section IV that increasing the number of jointly encoded subcarriers leads to the increase 


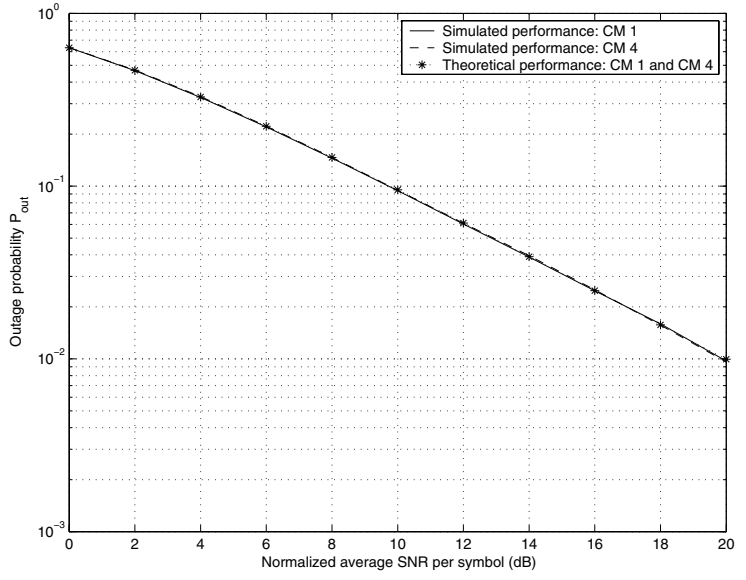

(a) No coding across subcarriers

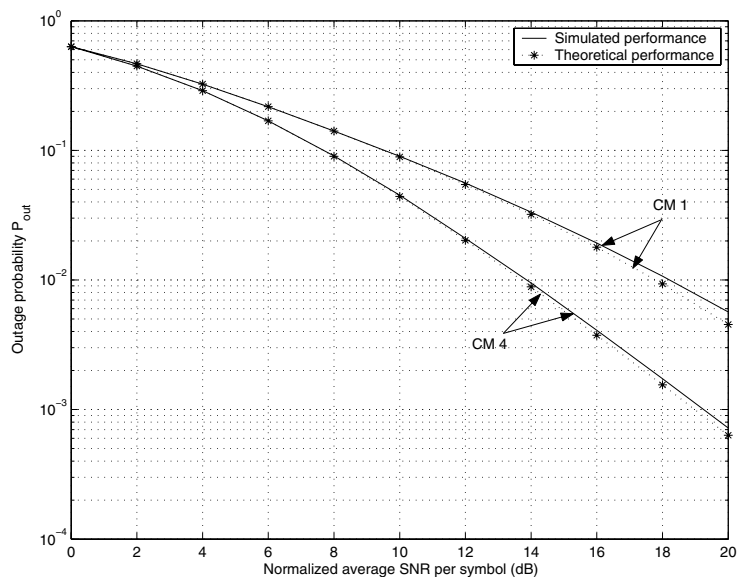

(b) Jointly encoding across two subcarriers

Fig. 3: Outage probability of coded multiband UWB system.

in the diversity gain, but the loss in the coding advantage.

\section{Vi. CONCLUSIONS}

In this paper, we provide the PEP and outage probability analysis that captures the unique multipath-rich and randomclustering characteristics of UWB channels. Both theoretical and simulation results reveal that the performances of uncoded multiband UWB systems do not depend on the clustering property, while the performances of coded multiband systems depend heavily on the correlations in the channel frequency response among different subcarriers, which in turn relate to the cluster arrival rate, the ray arrival rate, and the cluster and ray decay factors. In case of jointly coding across two subcarriers, we can draw some interesting conclusions as follows. When the product of the cluster arrival rate and cluster decay factor is small, e.g., in a short-range (0-4 meters) lineof-sight scenario, the effect of the first cluster will dominate and the UWB performance can be well approximated by taking into consideration only the first cluster. In contrast, when the product of the ray arrival rate and ray decay factor is much less than one, the performance seriously depends only on the first path in each cluster. The analysis for UWB-MIMO systems discloses that the coding gain strongly relates to the channel model parameters. The diversity gain on the other hand, can be improved by increasing the number of jointly encoded

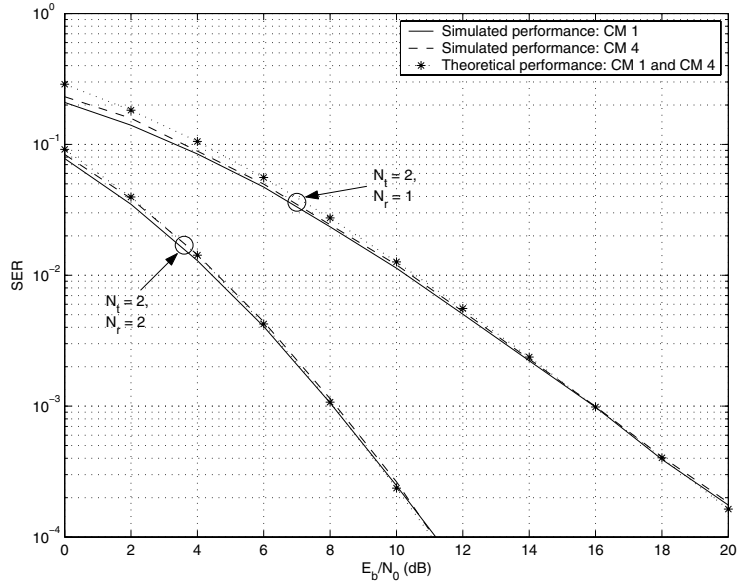

(a) Jointly encoding across two subcarriers

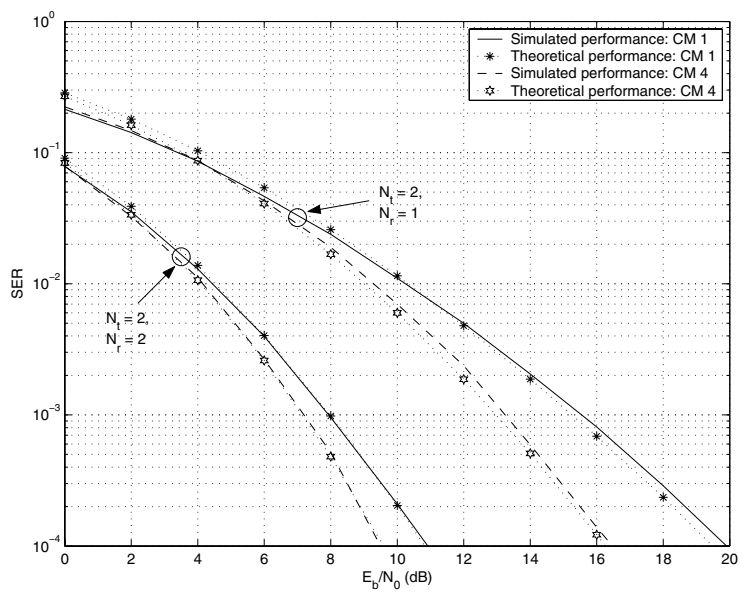

(b) Jointly encoding across four subcarriers

Fig. 4: PEP of multiband UWB-MIMO system.

subcarriers, the number of jointly encoded OFDM symbols, or the number of antennas, regardless of the random-clustering behavior of UWB channels.

\section{REFERENCES}

[1] J. Foerster, et. al, "Channel Modeling Sub-committee Report Final," IEEE802.15-02/490, Nov. 18, 2003.

[2] T. Kaiser, Ed., UWB Communications Systems - A Comprehensive Overview, EURASIP Series on Signal Processing and Communications, Hindawi, to appear.

[3] J. G. Proakis, Digital Communications, $4^{\text {th }}$ Ed., McGraw-Hill, New York, 2001.

[4] A. A. M. Saleh and R. A. Valenzuela, "A Statistical Model for Indoor Multipath Propagation," IEEE J. on Selected Areas in Commun., vol. 5, no. 2, pp. 128-137, Feb. 1987.

[5] IEEE 802.15WPAN High Rate Alternative PHY Task Group 3a (TG3a). Internet: www.ieee802.org/15/pub/ TG3a.html

[6] A. Batra, et. al, "Multi-Band OFDM Physical Layer Proposal for IEEE 802.15 Task Group 3a," IEEE P802.15-03/268r3, Mar. 2004.

[7] R. A. Horn and C. R. Johnson, Matrix Analysis, Cambridge Univ. Press, New York, 1985.

[8] M. K. Simon and M. S. Alouini, Digital Communication over Fading Channels: A Unified Approach to Performance Analysis, John Wiley and Sons, New York, 2000.

[9] A. M. Mathai and S. B. Provost, Quadratic Forms in Random Variables: Theory and Applications, Marcel Dekker Inc., New York, 1992.

[10] W. Su, Z. Safar, and K. J. R. Liu, "Towards Maximum Achievable Diversity in Space, Time and Frequency: Performance Analysis and Code Design," IEEE Trans. on Wireless Commun., vol. 4, no. 4, pp. 1847-1857, Jul. 2005.

[11] S. Alamouti, "A Simple Transmit Diversity Technique for Wireless Communications," IEEE J. on Selected Areas in Commun., vol. 16, no. 8, pp. 1451-1458, Oct. 1998. 